\title{
The Additive Value of Semi-Quantitative Analysis of 18F-FDG PET/CT For The Diagnosis of Device Related Infections In Patients With A Left Ventricular Assist Device.
}

Derk ten Hove ( $\nabla$ d.ten.hove@umcg.nl )

University Medical Center Groningen https://orcid.org/0000-0002-0717-6530

Ali R. Wahadat

Erasmus University Medical Center

Riemer H.J.A. Slart

University Medical Center Groningen

Marjan Wouthuyzen-Bakker

University Medical Center Groningen

Gianclaudio Mecozzi

University Medical Center Groningen

Kevin Damman

University Medical Center Groningen

Hester Witteveen

University Medical Center Groningen

\section{Kadir Caliskan}

Erasmus University Medical Center

Olivier C. Manintveld

Erasmus University Medical Center

\section{Bhanu Sinha}

University Medical Center Groningen

Ricardo P.J. Budde

Erasmus University Medical Center

Andor W.J.M. Glaudemans

University Medical Center Groningen

\section{Research Article}

Keywords: LVAD, device related infection, 18F-FDG-PET/CT, semi-quantitative analysis

Posted Date: October 21st, 2021 
DOl: https://doi.org/10.21203/rs.3.rs-990449/v1

License: (c) (1) This work is licensed under a Creative Commons Attribution 4.0 International License. Read Full License 


\title{
The additive value of semi-quantitative analysis of ${ }^{18} \mathrm{~F}-\mathrm{FDG}$ $\mathrm{PET} / \mathrm{CT}$ for the diagnosis of device related infections in patients with a Left Ventricular Assist Device.
}

Running Title: SQ analysis FDG-PET/CT for LVAD DRI

\author{
Derk ten Hove ${ }^{1,2}$, Ali R. Wahadat ${ }^{3,4,5}$, Riemer H.J.A. Slart ${ }^{1,6}$, Marjan Wouthuyzen-Bakker², \\ Gianclaudio Mecozzi ${ }^{7}$, Kevin Damman ${ }^{8}$, Hester Witteveen ${ }^{8}$, Kadir Caliskan ${ }^{4}$, Olivier C. \\ Manintveld ${ }^{4}$, Bhanu Sinha ${ }^{2}$, Ricardo P.J. Budde ${ }^{3 *}$, Andor W.J.M. Glaudemans ${ }^{1 *}$ (* shared \\ authorship)
}

1. University of Groningen, Department of Nuclear Medicine and Molecular Imaging, University Medical Center Groningen, Groningen, The Netherlands

2. University of Groningen, Department of Microbiology and Infection Prevention, University Medical Center Groningen, Groningen, The Netherlands

3. Department of Radiology and Nuclear Medicine, Erasmus University Medical Center, Rotterdam, the Netherlands

4. Department of Cardiology, Erasmus University Medical Center, Rotterdam, the Netherlands

5. Department of Cardiology, HagaZiekenhuis, The Hague, the Netherlands

6. Department of Biomedical Photonic Imaging, Faculty of Science and Technology, University of Twente, Enschede, the Netherlands

7. University of Groningen, Department of Cardiothoracic Surgery, University Medical Center Groningen, Groningen, The Netherlands

8. University of Groningen, Department of Cardiology, University Medical Center Groningen, Groningen, The Netherlands

Corresponding (also first) author: D. ten Hove: d.ten.hove@umcg.nl. Department of Nuclear Medicine and Molecular Imaging and the Department of Medical Microbiology and Infection Prevention, University Medical Center Groningen, Hanzeplein 1, 9713GZ, Groningen, Groningen, the Netherlands. Email: d.ten.hove@umcg.nl, Tel: +31629304806 Orcid ID: 0000-0002-0717-6530.

Word count: 5519

Financial support: This work was supported in part by an unconditional grant from PUSH: a collaboration between Siemens Healthineers and the UMCG. The sponsor had no role in the conceptualization, interpretation of findings, writing or publication of the article. KD reports speaker fees from Abbott, manufacturer of HMII and HM3 LVAD. All other authors declare no potential conflict of interest. 


\section{Abstract}

Background: Left ventricular assist devices (LVADs) improve quality of life and long-term survival in advanced heart failure, but device related infections (DRI) remain cumbersome. We evaluated the diagnostic capability of FDG-PET/CT and the additive value of semi-quantitative analysis for the diagnosis of DRI.

Methods: LVAD recipients undergoing FDG-PET/CT between December 2012 and August 2020 for suspected DRI were retrospectively included. FDG-PET/CT was performed and evaluated according to EANM guidelines and assessors were blinded to the clinical context of included patients. Final clinical diagnosis of driveline infection and/or central device infection, based on multidisciplinary consensus and findings during surgery whenever performed, was used as the reference for the diagnosis.

Results: 44 patients were evaluated for 62 episodes of suspected DRI. Clinical evaluation established driveline infection in 33 (54\%) episodes, central device infection in 8 (14\%) and combined infection in 2 (4\%). Visual analysis of FDG-PET/CT achieved a sensitivity and specificity of 0.83 and 0.73 , respectively, for driveline infections, while semi-quantitative analysis found comparable results $(p=0.77)$. For central device component infections, visual analysis of FDG-PET/CT achieved a sensitivity and specificity of 1.0 and 0.26 , respectively, while semi-quantitative analysis using SUVratio (background liver) achieved a sensitivity and specificity of 1.0 and 0.8 , respectively. Both SUVmax and SUVratio outperformed visual analysis $(p<0.001)$.

Conclusions: FDG-PET/CT is a valuable tool for the assessment of DRI in LVAD recipients. Semiquantitative analysis significantly increases specificity of FDG-PET/CT for the analysis of central device components and should be considered in equivocal cases after visual analysis.

Key words: LVAD, device related infection, ${ }^{18} \mathrm{~F}-\mathrm{FDG}-\mathrm{PET} / \mathrm{CT}$, semi-quantitative analysis

Abbreviations: CT: computed tomography, EANM: European Association of Nuclear Medicine, EARL: EANM research Itd., FDG: $\left[{ }^{18} \mathrm{~F}\right]$-fluorodeoxyglucose, IQR: interquartile range, LVAD: left ventricular assist device, PET: positron emission tomography, SUV max: maximum standardized uptake value, SUV ratio: standardized uptake value ratio 


\section{Full-text}

\section{Introduction}

Left ventricular assist device (LVAD) therapy increases survival and quality of life in patients with advanced heart failure, with median 5-year survival now approaching 50\% [1]. However, device related infections (DRI) occur frequently, with an incidence of $18.1 \%$ during the first year after implantation and $11.9 \%$ per year in the following years [1]. Establishing the presence, extent and the severity of DRI, especially in deep and central infections, can be difficult as conventional imaging modalities such as echocardiography and CT are hampered by device-related artefacts [2,3]. Most infections occur at the site of the driveline exit from the abdominal wall. These driveline infections have the potential to become life-threatening in case the infection reaches the central device components (pump housing, inflow tract and outflow tract), especially when this is complicated by device-related bloodstream infection, which is accompanied by an in-hospital mortality rate of up to 50\% [4-6]. Simultaneously, complicated device related infections can form a contraindication for heart transplantation, further increasing the risk of an unfavourable patient outcome.

Several studies assessed the diagnostic performance of $\left[{ }^{18} \mathrm{~F}\right]$-fluorodeoxyglucose positron emission tomography combined with low dose computed tomography (FDG-PET/CT) for diagnosing driveline and/or central LVAD component infections [7-15] and two meta-analyses combined their results $[15,16]$. Visual analysis of FDG-PET/CT has a high sensitivity for establishing LVAD infections and a high but variable specificity, with a pooled sensitivity of 0.95 (95\% confidence interval $(\mathrm{Cl}) 0.89-0.97)$ and a pooled specificity of $0.91(95 \% \mathrm{Cl} 0.54-$ 0.99). The variable specificity may be caused by differences in patient selection, scan interpretation or specific technical issues. Furthermore, semi-quantitative analysis might increase the specificity of FDG-PET/CT. So far, only one study investigated the role of semiquantitative analysis for diagnosing infections of both driveline and central LVAD components [8], while two studies focused on driveline infections alone $[7,10]$. The results of these studies were mixed. Therefore, we evaluated whether (1) factors that may affect FDG-PET/CT quality, such as patient preparation using a high-fat, low-carbohydrate (HFLC) diet and antibiotic use prior to PET/CT, affected visual analysis, and (2) whether quantification of FDG uptake around the LVAD and driveline improves the specificity and overall diagnostic accuracy of FDG-PET/CT. 


\section{Methods}

\section{Patients}

In this retrospective, dual-centre study, all consecutive patients that underwent FDG-PET/CT for assessment of suspected LVAD and/or driveline infections in the University Medical Center Groningen (UMCG) and Erasmus MC University Medical Center (EMC) were included from the start of using electronic patient files (first recorded case December 2012) until 31 August 2020. Initial suspicion of infection was based on clinical history and presentation. Presenting symptoms of patients were recorded alongside general demographic data and information about the LVAD; e.g. indication for implantation, type and brand of LVAD and the time interval between LVAD implantation and FDG-PET/CT. Findings from clinical investigations such as inflammatory markers, blood cultures, cultures from exit-site swabs and findings during surgery (if performed) were recorded, together with information on the use of intravenous antibiotics at the time of the FDG-PET/CT, including the duration in days. If patients underwent FDG-PET/CT scans during multiple episodes of suspected infection, the later scans were only included if there were recurring symptoms after a symptom-free period of at least 1 month and after treatment for the first episode was fully completed (minimum interval between consecutive FDG-PET/CTs was 2 months) and special care was taken to avoid FDG-PET/CT scans that were performed to establish treatment effect. The study was approved and the need for informed consent waived by the Local Medical Ethics Review Committees of both centres due to the non-WMO (Dutch law on studies involving human subjects) nature of this study using retrospective data: protocol nr M19.223017 (UMCG) / MEC-2019-0613 (EMC).

\section{FDG-PET/CT protocol}

The protocol for FDG-PET/CT preparation that was followed for each scan was documented. This included the duration of the fasting period before FDG-PET/CT, whether or not an HFLC diet had been used prior to the scan, patient blood glucose levels at the time of FDG injection, the injected FDG activity, and the used vendor type of PET/CT camera system.

\section{Final diagnosis}

Infections of the driveline and those of the LVAD pump housing were evaluated as two separate diagnoses since their FDG-PET/CT interpretation, treatment and prognosis are different. The possible outcomes were: absence of device related infection, driveline infection, infection of central device components and combined infection of both driveline 
and central device components. In case surgery was performed, macroscopic signs of infection during surgery and evidence from cultures and molecular diagnostics on swabs in the affected areas or from removed tissues were considered as the gold standard for the diagnosis. If surgery was not performed, the final clinical diagnosis was established by the treating physician within the multidisciplinary LVAD team, which included cardiologists, thoracic surgeons, engineers specialized in LVAD technique and maintenance, medical microbiologists and infectious disease specialists, with radiologists and nuclear medicine physicians consulted on case-by-case basis. All clinical findings, including swabs for driveline exit sites, blood cultures, findings from imaging (including PET) and the outcome during a minimum of 4 months of follow-up, were combined to a post-hoc composite gold standard, an approach similar to that often used for the diagnosis of endocarditis $[17,18]$. In 7 cases the final diagnosis of either driveline or central device component infection remained uncertain even after obtaining all other available clinical information and follow-up. These cases with an indeterminate final diagnosis were excluded from FDG-PET/CT diagnostic accuracy analyses.

\section{Visual analysis}

FDG-PET/CT visual analysis and standardized uptake value (SUV) calculations were performed using Syngo.via VB30 (Siemens Healthineers, Knoxville, TN, USA). Scans were analysed through consensus reading by two experienced nuclear medicine physicians (AG and RS), both of whom were blinded to the clinical context of the patients. Visual evaluation and interpretation of FDG-PET/CT was performed according to EANM guidelines and was based on the FDG uptake pattern, intensity and extension of any lesions showing increased FDG-uptake around the driveline and/or the central LVAD components, including nearby soft tissue lesions and fluid collections. Both attenuation-corrected and uncorrected images were used for the analyses [19].

\section{Semi-quantitative analysis}

After delineation of volumes of interest (VOI) the SUVmax and SUVmean were measured at 6 predefined areas of interest in each patient. These regions comprised three areas alongside the peripheral driveline tract (driveline exit site, suprafascial driveline tract, and subfascial driveline) and 3 areas around the central device components (intrathoracic driveline, inflow canula / pump housing, and the outflow tract) (Figure 1 and 2). Although the pump-pocket of the Heartmate II is technically located abdominally, we defined it as a central device 
component because of its direct connection to inflow and outflow tracts, and the clinical severity when the pocket is infected. The highest SUVmax value alongside the driveline and the highest value around the central device components were used for establishing the diagnostic accuracy of semi-quantitative analysis.

After delineation of reference regions in the thoracic aorta and liver, the SUVratio was calculated by dividing the highest SUVmax value alongside the driveline and the highest SUVmax value around the central device components by the mean activity in both reference regions separately. The aorta was carefully delineated to exclude the aortic vascular wall, and it was verified that the liver function tests were normal at the time of FDG-PET/CT in the included patients. All calculations were performed on EANM research Itd (EARL) reconstructed images [18].

\section{Potential confounders}

Factors that could potentially affect the assessment of either driveline infection or infections of central device components were identified for further analysis. These included patient age, gender, BMI, diabetes mellitus, the indication for LVAD implantation (ischemic or dilated cardiomyopathy), type of LVAD (either Heartmate II or Heartmate 3), type of PET/CT system, patient preparation using a high-fat low carbohydrate (HFLC) diet and the duration of antibiotic use prior to FDG-PET/CT.

\section{Statistics}

For demographic data, continuous variables are presented as mean +/- SD, while categorical variables are reported as frequencies. We used logistic regression to evaluate the accuracy of visual analysis and semi-quantitative analysis diagnosis driveline and/or central device infections. Potential confounders were tested using univariate logistic regression for effect on visual analysis and final diagnosis. Multivariate logistic regression was not performed on account of insufficient case numbers. For semi-quantitative analyses receiver operating characteristics (ROC) analysis was performed to determine the optimum threshold values to maximize sensitivity and specificity. For all statistical analyses a two-tailed P-value $\leq 0.05$ was considered statistically significant. Analyses were performed using IBM ${ }^{\circledR}$ SPSS 26 [IBM Corp]. 


\section{Results}

\section{Patient characteristics}

In total, 44 patients underwent a total of 70 FDG-PET/CT scans because of a clinical suspicion of LVAD and/or driveline infection. Eight scans were excluded because they were follow-up scans performed to establish treatment effect, leaving 62 scans for analysis. Of these, 20 patients ( 25 scans) were evaluated in the EMC, while the remainder of 24 patients ( 37 scans) were evaluated in UMCG. The indication for LVAD implantation was non-ischemic cardiomyopathy in 25 of the 44 patients (57\%) and ischemic cardiomyopathy in the remaining $19(43 \%)$ patients. The implanted LVAD system was either the Heartmate 3 (32/44 or $73 \%$ of patients) or the Heartmate II (12/44 or $27 \%$ of patients). For an overview of demographic data see Table 1.

\section{FDG-PET/CT protocol}

Preparation for the FDG-PET/CT was different for the two participating centres: While in both centres a fasting period of at least 6 hours was standard, in the UMCG the 24-hour high-fat low-carbohydrate diet was only formalized at the beginning of 2020, while in the EMC this had been the standard since October 2016 [14]. The HFLC diet was used to prepare patients for FDG-PET/CT in 28 of 62 scans (45\%). In 27 instances, use of this diet could not be verified. The PET/CT systems used included the Siemens Biograph mCT40, mCT64 and the Biograph 128 VISION (Siemens Healthineers, KN, USA) for 16, 20 and 26 scans respectively. FDG-PET/CT was in both centres performed 60 minutes after injection of FDG. The average injected activity was $263 \mathrm{MBq}$ or $2.9 \mathrm{MBq} / \mathrm{kg}$ per patient, in accordance to EANM guidelines [20].

\section{Final diagnosis}

As mentioned earlier, for the final diagnosis of a device specific infection a distinction was made between driveline and central device related infections. Driveline infection was confirmed in 33 of 61 (54\%) cases, while in one additional case the diagnosis could neither be confirmed nor excluded and the patient was treated pragmatically with antibiotics. Isolated infection of central device components was established in 8 of 56(14\%) episodes, with an additional 6 indeterminate cases that were also treated empirically. Two patients (4\%) had a combined infection of both driveline and central device components. See also table 1. 


\section{Visual analysis}

Visual analysis of FDG-PET/CT reliably predicted the presence of driveline infections (OR: 13.1 [95\% Cl: 3.8-45.1]; $p<0.001$ by logistic regression). The corresponding sensitivity and specificity were 0.83 and 0.73 , respectively. For visual analysis of suspected central device component infections, logistic regression could not be performed due to non-convergence, caused by the absence of any false negative findings, which invalidates the logistic regression model. Further analysis using cross-tabs showed that visual analysis suffered from low specificity. While the diagnosis of central device component infection was rejected in all 10 cases that were classified as negative based on visual analysis, leading to a sensitivity of 1.0, of the remaining 46 cases that were classified as positive, 34 were false positives, resulting in a specificity of only 0.26. Analysis of FDG-uptake patterns showed that heterogeneous FDG-uptake and even (multi)focal uptake around the central device components were not always predictive of infection: of the 24 patients with heterogeneous uptake in this area, only 4 were diagnosed with central device component infection and of the 14 patients with (multi)focal uptake, only 6 had infection of the central device components. Non-attenuation corrected images showed a similarly limited specificity: increased uptake was shown in 24 (43\%) patients, but infection was present in only $6(25 \%)$ of them. However, this was higher than in the group of $28(57 \%)$ patients in whom NAC activity was not increased. Of this group, 4 (14\%) had a central device component infection.

\section{Semi-quantitative analysis}

Semi-quantitative analysis using SUVmax reliably predicted driveline infections (OR: 3.97 [95\% $\mathrm{Cl}: 1.79-8.81], \mathrm{p}<0.001$; by logistic regression). ROC curve analysis showed that the optimum balance between sensitivity and specificity was achieved using a SUVmax cut-off value of 3.53, resulting in a sensitivity and specificity of 0.94 and 0.80 , respectively. This constituted an improvement compared to visual analysis from 0.79 to 0.88 , but the difference was not statistically significant $(p=0.77)$. For diagnosis of infection of central device components semiquantitative analysis using SUVmax reliably predicted infection (OR 1.85 [95\% Cl: 1.17-2.92], $\mathrm{p}=0.009$; by logistic regression) as did SUVratio using the thoracic blood pool as reference region (OR: 3.66 [95\% Cl: 1.44-9.29] p=0.006; by logistic regression) and SUVratio using the liver as reference region (OR: 12.99 [95\% Cl: 2.23-75.78] $p=0.004$; by logistic regression). Optimal sensitivity and specificity was achieved using the SUVratio with the liver as a reference 
region. At a SUVratio cut-off value of 2.45 the corresponding sensitivity and specificity were 1.00 and 0.80 , respectively. Using the thoracic blood pool also achieved good sensitivity and specificity. At a cut-off value of 3.04 these were 0.89 and 0.83 , respectively. SUVmax alone reached its optimum at a cut-off value of 5.14, achieving a sensitivity and specificity of 0.67 and 0.80 , respectively. The improvements of sensitivity and specificity compared to visual analysis were statistically significant: $p<0.001$, regardless of the semi-quantitative method used. The corresponding ROC curves for both driveline and central device components are shown in Figure 3.

\section{Combined visual / semi-quantitative analysis}

Because visual analysis showed high negative predictive value for central device component infection, semiquantitative methods were also evaluated after excluding all cases that were deemed negative based on visual analysis alone. Again, logistic regression showed predictive values for SUVmax (OR: 1.73 [95\% Cl: 1.11-2.69], p=0.016); SUVratio with the thoracic aorta bloodpool as background (OR: 3.26 [95\% $\mathrm{Cl}: 1.30-8.14], \mathrm{p}=0.012$ ); and SUVratio using the liver as background (OR: 11.80 [95\% $\mathrm{Cl}: 1.92-72.36], \mathrm{p}=0.008$ ). Optimal cut-off values were unchanged for all metrics and again the highest sensitivity and specificity was achieved by using the SUVratio with the liver as background: 1.00 and 0.79 , respectively.

\section{Potential confounders for FDG-PET/CT}

Of the evaluated potential confounders, only the duration of intravenous antibiotics use and the interval between LVAD implantation and FDG-PET/CT showed a statistically significant effect on visual analysis of FDG-PET/CT. A longer duration of antibiotic use was associated with a lower chance of finding central device component infection (OR: 0.99 [95\% Cl: 0.98-1.00] per day of antibiotic use, $p=0.04$ by logistic regression). Antibiotic use did not affect the final diagnosis of either driveline infection or infection of central device components (OR: 1.00 [95\% $\mathrm{Cl}: 0.99-1.01], p=0.233$; by logistic regression), implying a confounding effect. Considering the time interval between LVAD implantation and FDG-PET/CT, the scan was performed within 30 days after implantation in only two cases. Both were correctly identified as negative for driveline infection and infection of central device components by both visual analysis and semiquantitative analysis. Twelve scans were performed within 90 days after LVAD implantation. In this group the risk of driveline infection was unexpectedly lower than in the 50 FDG-PET/CT scans performed 90 days or more after LVAD implantation: $27 \%$ vs 64\%; 
OR 4.74 [95\% Cl: 1.11-20.2], p=0.04 by logistic regression, while the chance of finding driveline infection by FDG-PET/CT in this group was not significantly increased: OR: 2.49 [95\% $\mathrm{Cl}: 0,69$ 8,99], $p=0.16$.

Other potential confounders did not significantly affect FDG-PET/CT accuracy by univariate logistic regression. These included patient age, gender, BMI, diabetes, indication for LVAD implantation, LVAD type, PET/CT system and use of an HFLC diet. Interestingly, increasing age was associated with a lower risk of driveline infection (OR: 0.93 per year [95\% $\mathrm{Cl}$ : 0.89-0.99], $p=0.02$ ), without a confounding effect on FDG-PET/CT accuracy. For a full overview see Supplemental data.

\section{Discussion}

In this multicentre retrospective study the diagnostic accuracy of FDG-PET/CT for LVAD device related infections (DRI) was evaluated in 44 patients and 62 episodes of suspected infection, making it one of the largest cohorts for this indication to date [7-15]. Additionally, we specifically stratified for driveline infections and infections involving central device components, the assessors were fully blinded to the clinical context of patients and used both $A C$ and NAC images. Furthermore, we included semi-quantitative analyses at predefines areas and performed in accordance with EARL recommendations.

FDG-PET/CT showed good sensitivity and specificity for diagnosing infections of the driveline, both on visual and semi-quantitative analysis, which is in line with current literature [7-15]. Visual analysis also showed good sensitivity for diagnosing a central device infection. However, specificity was extremely poor. Semi-quantitative analysis clearly outperformed visual analysis for this specific indication, with best results achieved using the liver as the reference region: specificity of FDG-PET/CT improved from 0.26 to 0.80 in central device compartments. By contrast, its high sensitivity remained unchanged at 1.0, compared with visual analysis, and this improvement was statistically significant. These results indicate that in all cases in which infection of central device components cannot be ruled out based on visual analysis, semi-quantitative analysis may significantly increase the diagnostic accuracy.

There are multiple explanations for the low specificity of visual analysis of FDG-PET/CT for central device components [21]. Firstly, in our study the assessors were blinded to the patients' clinical context, while in about half of earlier studies, assessors were not (fully) 
blinded, which may have led to observer bias. Secondly, many patients in our study had a heartmate 3 and distinguishing between sterile inflammation at the insertion of the pump in the left ventricle proved difficult by visual analysis, even while using NAC images. Another explanation for the low specificity and the heterogenous uptake in many patients could be that the outflow tract for both Heartmate II and 3 consists of polyethylenterephthalat (PET; Dacron ${ }^{\circledR}$ ), a synthetic material known to cause reactive FDG uptake on FDG-PET/CT in up to $97 \%$ of Dacron ${ }^{\circledR}$ vascular graft recipients [21]. This increased uptake can be heterogeneous in up to $30 \%$ of patients and remain unchanged for at least 16 years. Therefore, a follow-up study to assess the value of baseline FDG-PET/CT after LVAD implantation would be of importance, since baseline scans could potentially help distinguish sterile inflammation from infectious processes, increasing the discriminative power of FDG-PET/CT.

\section{Recommendation for implementation of FDG-PET/CT in suspected DRI in LVAD patients}

Based on the findings in this study, visual and semi-quantitative analysis of FDG$\mathrm{PET} / \mathrm{CT}$ can be complementary methods for the evaluation of driveline infections and central device component infections. We propose a flow chart for deciding which method of assessment is most suitable for different parts of the evaluation in Figure 4. Future prospective studies would be invaluable for further validation of the cut-off values we found for semiquantitative analysis and to more precisely establish the role of HFLC diet and the use of nonattenuation corrected images.

\section{Limitations}

This study had a retrospective design which makes it prone to inclusion bias, though care was taken to include every consecutive patient with a suspicion of infection. Another limitation concerns the gold standard for the diagnosis: direct culture and molecular diagnostics using 16S PCR of the suspected device parts. This gold standard for the diagnosis is frequently unattainable because surgery is not always performed and other methods of retrieving the target tissues (e.g. CT-guided biopsy) are only very rarely used. While exit site swabs and blood cultures can give an indication for device infection, they are often insufficient to fully ascertain the diagnosis, especially considering infections of the central device components. To minimize the risk of incorporation bias, findings during surgery were considered as the true gold standard for the diagnosis whenever available, and when these were unavailable, all clinical findings, such as blood cultures, driveline exit site swabs, results from other available imaging 
modalities and outcomes during follow-up were considered together as a composite gold standard to avoid reliance on FDG-PET/CT alone.

The effect of (non-)adherence to the HFLC diet on FDG-PET/CT accuracy could not be statistically verified in our study, likely due to insufficient case numbers in this group as only 4 patients with a HFLC diet had a final diagnosis of central device component infection. Because physiological myocardial uptake leads to decreased evaluability of the left ventricle wall and because central device component infections have a well-known risk of concurrent endocarditis, we still recommend using this diet for all cases of suspected LVAD infection.

\section{Conclusion}

FDG-PET/CT is a valuable tool for establishing the diagnosis of DRI in LVAD recipients, including both driveline and central device component infections. Visual analysis and semi-quantitative analysis both reliably establish presence of driveline infections. For central device components, visual analysis suffers from poor specificity. For this indication, semi-quantitative analysis significantly increases FDG-PET/CT diagnostic accuracy.

\section{Compliance with ethical standards}

Financial support: This work was supported in part by an unconditional grant from PUSH: a collaboration between Siemens Healthineers and the UMCG. The sponsor had no role in the conceptualization, interpretation of findings, writing or publication of the article. KD reports speaker fees from Abbott, manufacturer of HMII and HM3 LVAD. All other authors declare no potential conflict of interest.

Ethics approval: Informed consent was waived by the medical ethics review boards of both medical centres, as the study was retrospective, non-WMO (Dutch law on studies involving human subjects) designated research: protocol nr M19.223017 (UMCG) / MEC-2019-0613 (EMC). 


\section{References}

1. Molina EJ, Shah P, Kiernan MS, Cornwell WK 3rd, Copeland H, Takeda K, Fernandez FG, Badhwar V, Habib RH, Jacobs JP, KoehI D, Kirklin JK, Pagani FD, Cowger JA. The Society of Thoracic Surgeons Intermacs 2020 Annual Report. Ann Thorac Surg. 2021 Mar;111(3):778-792.

2. Lesicka A, Feinman JW, Thiele K, Andrawes MN. Echocardiographic artifact induced by HeartWare left ventricular assist device. Anesth Analg. 2015;120(6):1208-11.

3. Shroff GS, Ocazionez D, Akkanti B, Vargas D, Garza A, Gupta P, et al. CT Imaging of Complications Associated with Continuous-Flow Left Ventricular Assist Devices (LVADs). Semin Ultrasound, CT MRI [Internet]. 2017;38(6):616-28.

4. Kirklin JK, Naftel DC, Pagani FD, Kormos RL, Stevenson LW, Blume ED, et al. Seventh INTERMACS annual report: 15,000 patients and counting. J Hear Lung Transplant [Internet]. 2015;34(12):1495-504.

5. Aggarwal A, Gupta A, Kumar S, Baumblatt JA, Pauwaa S, Gallagher C, et al. Are blood stream infections associated with an increased risk of hemorrhagic stroke in patients with a left ventricular assist device? ASAIO J. 2012;58(5):509-13.

6. Trachtenberg BH, Cordero-Reyes AM, Aldeiri M, Alvarez P, Bhimaraj A, Ashrith G, et al. Persistent blood stream infection in patients supported with a continuous-flow left ventricular assist device is associated with an increased risk of cerebrovascular accidents. J Card Fail [Internet]. 2015;21(2):119-25.

7. Avramovic N, Dell'Aquila AM, Weckesser M, Milankovic D, Vrachimis A, Sindermann $J R$, et al. Metabolic volume performs better than SUVmax in the detection of left ventricular assist device driveline infection. Eur J Nucl Med Mol Imaging. 2017;44(11):1870-7.

8. Dell'Aquila AM, Mastrobuoni S, Alles S, Wenning C, Henryk W, Schneider SRB, et al. Contributory role of fluorine 18-fluorodeoxyglucose positron emission tomography/ computed tomography in the diagnosis and clinical management of infections in patients supported with a continuous-flow left ventricular assist device. Ann Thorac Surg. 2016;101(1):87-94.

9. Dell'aquila AM, Avramovic N, Mastrobuoni S, Motekallemi A, Wisniewski K, Scherer M, et al. Fluorine-18 fluorodeoxyglucose positron emission tomography/computed tomography for improving diagnosis of infection in patients on CF-LVAD: Longing for more insights'. Eur Heart J Cardiovasc Imaging. 2018;19(5):532-43.

10. Kanapinn P, Burchert W, Körperich H, Körfer J. 18F-FDG PET/CT-imaging of left ventricular assist device infection: a retrospective quantitative intrapatient analysis. J Nucl Cardiol. 2018;1-10.

11. Kim J, Feller ED, Chen W, Liang Y, Dilsizian V. FDG PET/CT for Early Detection and Localization of Left Ventricular Assist Device Infection: Impact on Patient Management and Outcome. JACC Cardiovasc Imaging. 2019;12(4):722-9.

12. Bernhardt AM, Pamirsad MA, Brand C, Reichart D, Tienken M, Barten MJ, et al. The value of fluorine-18 deoxyglucose positron emission tomography scans in patients with ventricular assist device specific infections. Eur J Cardio-thoracic Surg. 2017;51(6):1072-7.

13. De Vaugelade C, Mesguich C, Nubret K, Camou F, Greib C, Dournes G, et al. Infections in patients using ventricular-assist devices: Comparison of the diagnostic performance of 18 F-FDG PET/CT scan and leucocyte-labeled scintigraphy. J Nucl Cardiol. 2019;26(1):42-55. 
14. Akin S, Muslem R, Constantinescu AA, Manintveld OC, Birim O, Brugts JJ, et al. 18F-FDG $\mathrm{PET} / \mathrm{CT}$ in the diagnosis and management of continuous flow left ventricular assist device infections: A Case Series and Review of the Literature. ASAIO J. 2017

15. Tam MC, Patel VN, Weinberg RL, Hulten EA, Aaronson KD, Pagani FD, et al. Diagnostic Accuracy of FDG PET/CT in Suspected LVAD Infections. JACC Cardiovasc Imaging. 2019;1-12.

16. Ten Hove D, Treglia G, Slart RHJA, Damman K, Wouthuyzen-Bakker M, Postma DF, Gheysens O, Borra RJH, Mecozzi G, van Geel PP, Sinha B, Glaudemans AWJM. The value of 18F-FDG PET/CT for the diagnosis of device-related infections in patients with a left ventricular assist device: a systematic review and meta-analysis. Eur J Nucl Med Mol Imaging. 2020 Jun 27. doi: 10.1007/s00259-020-04930-8. Epub ahead of print. PMID: 32594196.

17. Swart LE, Gomes A, Scholtens AM, Sinha B, Tanis W, Lam MGEH, van der Vlugt MJ, Streukens SAF, Aarntzen EHJG, Bucerius J, van Assen S, Bleeker-Rovers CP, van Geel PP, Krestin GP, van Melle JP, Roos-Hesselink JW, Slart RHJA, Glaudemans AWJM, Budde RPJ. Improving the Diagnostic Performance of 18F-Fluorodeoxyglucose PositronEmission Tomography/Computed Tomography in Prosthetic Heart Valve Endocarditis. Circulation. 2018 Oct 2;138(14):1412-1427. doi: 10.1161/CIRCULATIONAHA.118.035032. PMID: 30018167.

18. Gomes A, Glaudemans AWJM, Touw DJ, van Melle JP, Willems TP, Maass AH, et al. Diagnostic value of imaging in infective endocarditis: a systematic review. Lancet Infect Dis. 2017;17(1):1-14.

19. Slart RHJA, Glaudemans AWJM, Gheysens O, Lubberink M, Kero T, Dweck MR, et al; 4Is Cardiovascular Imaging: a joint initiative of the European Association of Cardiovascular Imaging (EACVI); European Association of Nuclear Medicine (EANM). Procedural recommendations of cardiac PET/CT imaging: standardization in inflammatory-, infective-, infiltrative-, and innervation (4ls)-related cardiovascular diseases: a joint collaboration of the EACVI and the EANM. Eur J Nucl Med Mol Imaging. 2021 Apr;48(4):1016-1039.

20. Boellaard R, Delgado-Bolton R, Oyen WJG, Giammarile F, Tatsch, K, Eschner W, et al. FDG PET/CT: EANM procedure guidelines for tumour imaging: version 2.0. Eur J Nucl Med Mol Imaging. 2015;42(2):328-54.

21. Keidar Z, Pirmisashvili N, Leiderman M, Nitecki S, Israel O. 18F-FDG uptake in noninfected prosthetic vascular grafts: incidence, patterns, and changes over time. J Nucl Med. 2014 Mar;55(3):392-5. doi: 10.2967/jnumed.113.128173. Epub 2014 Feb 10. PMID: 24516259. 
Table 1. Overview patient characteristics

\begin{tabular}{|c|c|}
\hline \multicolumn{2}{|l|}{ Characteristics } \\
\hline Number of patients per medical center & \\
\hline Erasmus MC University Medical Center & 20 patients ( 25 episodes of suspected infection) \\
\hline University Medical Center Groningen & 24 patients (37 episodes of suspected infection) \\
\hline Age (mean, SD) Years & 55 years $(12.0)$ \\
\hline \multicolumn{2}{|l|}{ Gender } \\
\hline Male & $37(84 \%)$ \\
\hline Female & $7(16 \%)$ \\
\hline \multicolumn{2}{|l|}{ Indication LVAD } \\
\hline Dilated cardiomyopathy & $25(57 \%)$ \\
\hline Ischemic Heart failure & $19(43 \%)$ \\
\hline \multicolumn{2}{|l|}{ LVAD type $(n=44)$} \\
\hline Heartmate 3: & $32(73 \%)$ \\
\hline Heartmate II: & $12(27 \%)$ \\
\hline Days since LVAD implantation: median (IQR; range) & $\begin{array}{l}\text { Overall: } 267 \text { (IQR 136.5-617.5; range } 24-1548) \\
\text { Heartmate II: } 179 \text { (IQR 132-669; range } 30-1548) \\
\text { Heartmate 3: } 304 \text { (IQR } 138 \text { - 616; range } 24-1279 \text { ) }\end{array}$ \\
\hline \multicolumn{2}{|l|}{ HFLC diet $(n=62)$} \\
\hline Yes & $28(45 \%)$ \\
\hline No & $34(55 \%)$ \\
\hline \multicolumn{2}{|l|}{ Blood glucose level at PET/CT } \\
\hline$<11 \mathrm{mmol} / \mathrm{L}(198 \mathrm{mg} / \mathrm{dl})$ & $59(95 \%)$ \\
\hline$\geq 11 \mathrm{mmol} / \mathrm{L}$ & $3(6 \%)$ \\
\hline \multicolumn{2}{|l|}{ PET/CT type $(n=62)$} \\
\hline Biograph 40 (mCT) & $16(27 \%)$ \\
\hline Biograph 64 (mCT) & $20(32 \%)$ \\
\hline Biograph 128 (Vision) & $26(42 \%)$ \\
\hline \multicolumn{2}{|l|}{ Final diagnosis $(n=62)$} \\
\hline Driveline infected $(n=61)$ & $33(54 \%)$ \\
\hline Central device components infected $(n=56)$ & $8(14 \%)$ \\
\hline $\begin{array}{l}\text { Combined infection of driveline and central device } \\
\text { components }(n=55)\end{array}$ & $2(4 \%)$ \\
\hline
\end{tabular}




\begin{tabular}{|c|c|}
\hline \multicolumn{2}{|l|}{ Characteristics (continued) } \\
\hline Cultured pathogen & \\
\hline Driveline infection $(n=35)$ & \\
\hline Blood cultures & $\begin{array}{l}\text { S. aureus (3), E. cloacae (2), E. faecium (1), C. acnes } \\
\text { (1), S. dysgalactiae (1), negative (27) }\end{array}$ \\
\hline Cultures exit site / removed tissue & $\begin{array}{l}\text { S. aureus (17), P. aeruginosa (4), E. cloacae (2), } H \text {. } \\
\text { parainfluenzae (1), K. oxytoca (1), S. hominis (1), M. } \\
\text { chelonae (1), P. mirabilis (1), S. dysgalactiae (1), } \\
\text { negative (6) }\end{array}$ \\
\hline Central device component infection $(n=10)$ & \\
\hline Blood cultures & $\begin{array}{l}\text { CNS (2), E. faecalis (2), S. aureus (2), S. lugdunensis } \\
(1) \text {, negative (3) }\end{array}$ \\
\hline Cultures of removed tissue & $\begin{array}{l}\text { S. aureus (2), H. influenzae \& S. epidermidis (1), S. } \\
\text { epidermidis \& C. acnes \& Candida (1), P. mirabilis (1), } \\
\text { negative (5) }\end{array}$ \\
\hline
\end{tabular}


Figure 1. Areas of interest alongside driveline and at central device components (no device specific infection)

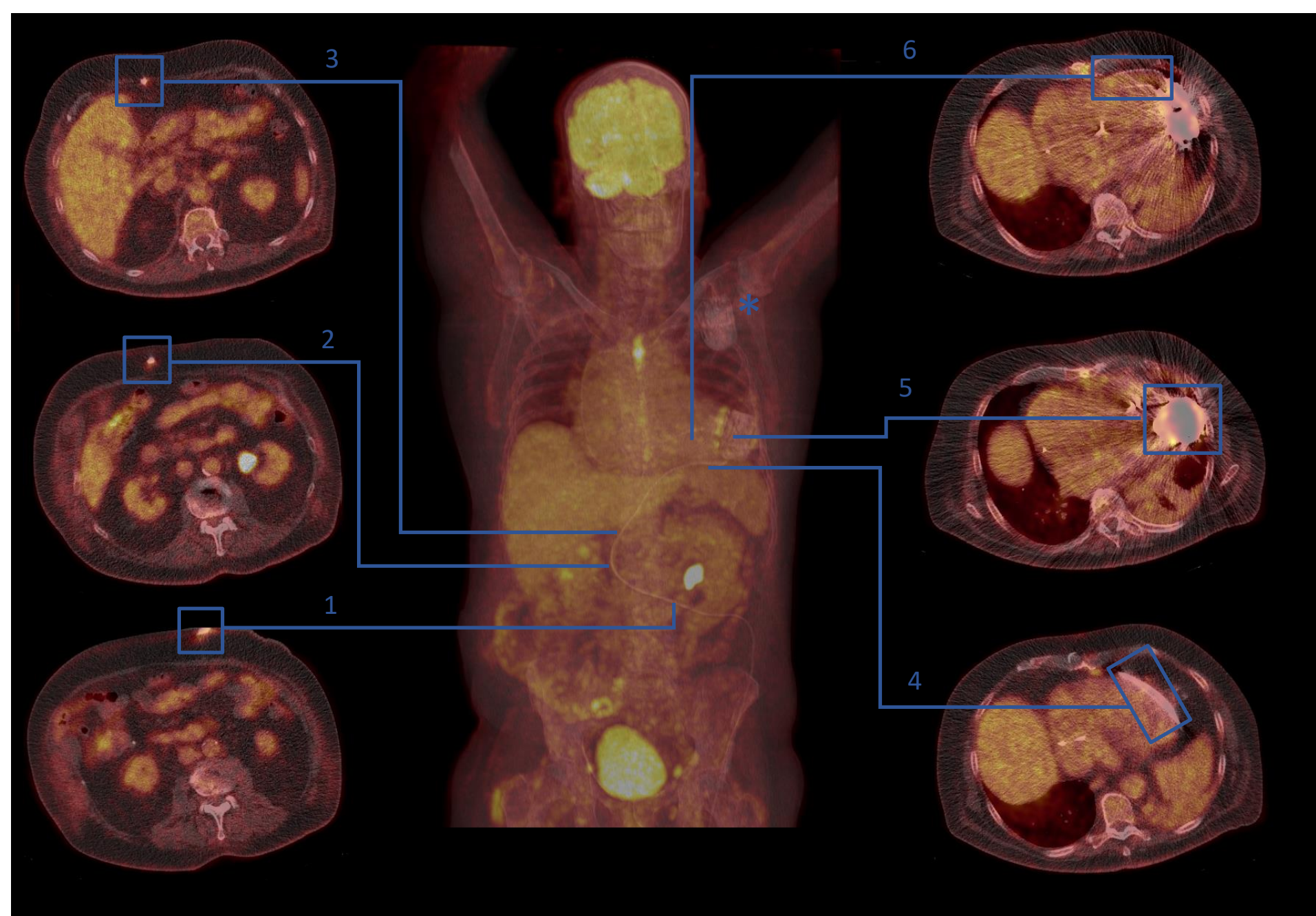

Legend: 1: Driveline exit site, 2: Suprafascial driveline, 3: Subfascial driveline, 4: Intrathoracic part driveline 5: Pump housing, 6: Outflow tract. Note also the ICD $\left({ }^{*}\right)$ and the increased FDG uptake at the sternum. In absence of local symptoms, this finding was explained as postoperative reactive uptake (complicated LVAD implantation 6 months before) 
Figure 2. Areas of interest alongside LVAD driveline and at central device components (both infected)

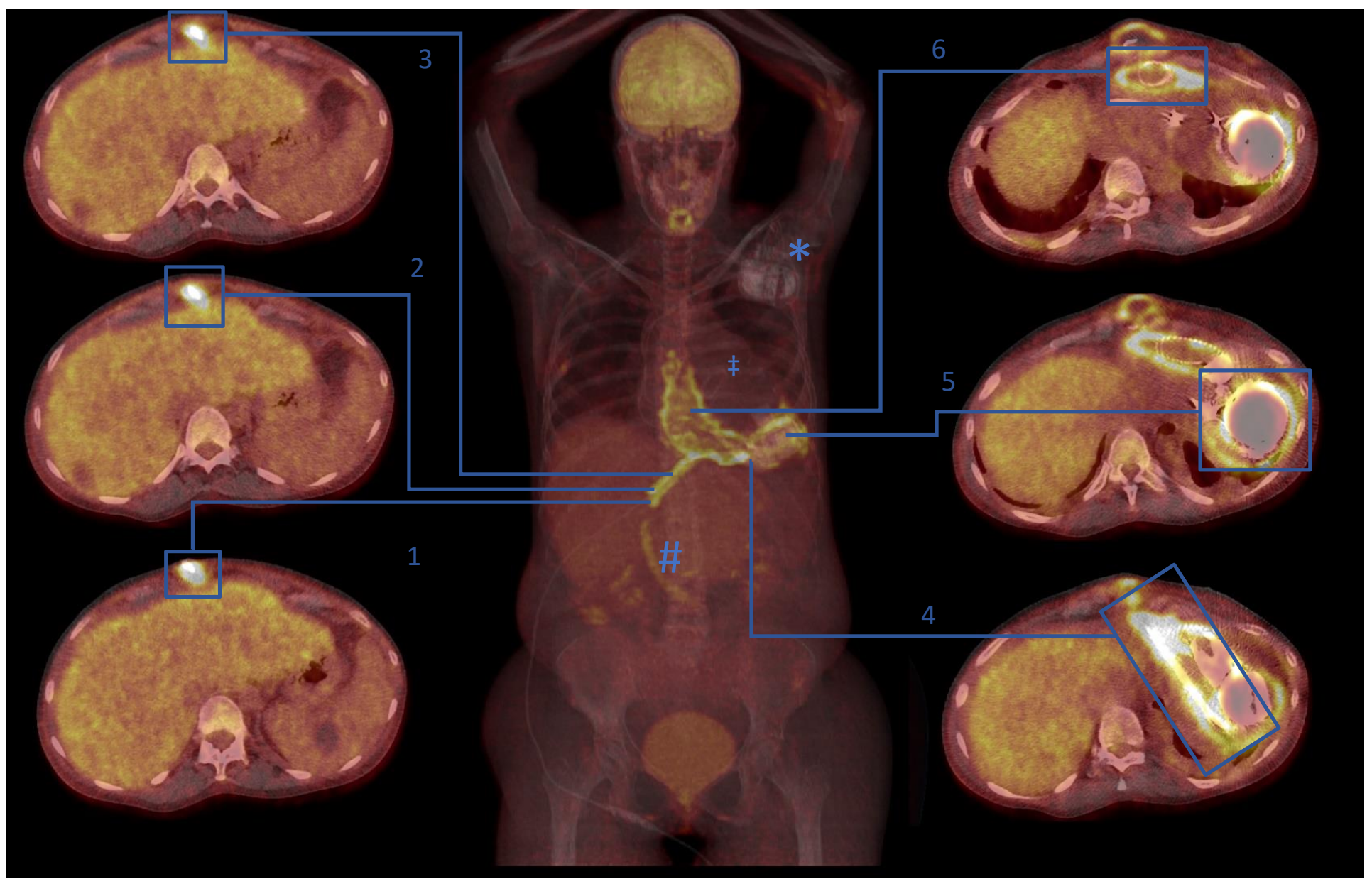

Legend: 1: Driveline exit site, 2: Suprafascial driveline, 3: Subfascial driveline, 4: Intrathoracic part driveline 5: Pump housing, 6: Outflow tract. NB: Note also the ICD $(*)$, an MVP ring without signs of infection ( $¥)$ and the residual FDG uptake below the insertion of the driveline after surgical debridement and shortening of the driveline 2 months before (\#). 
Figure 3. ROC curves FDG-PET/CT for infection of the LVAD driveline and central device components

Driveline

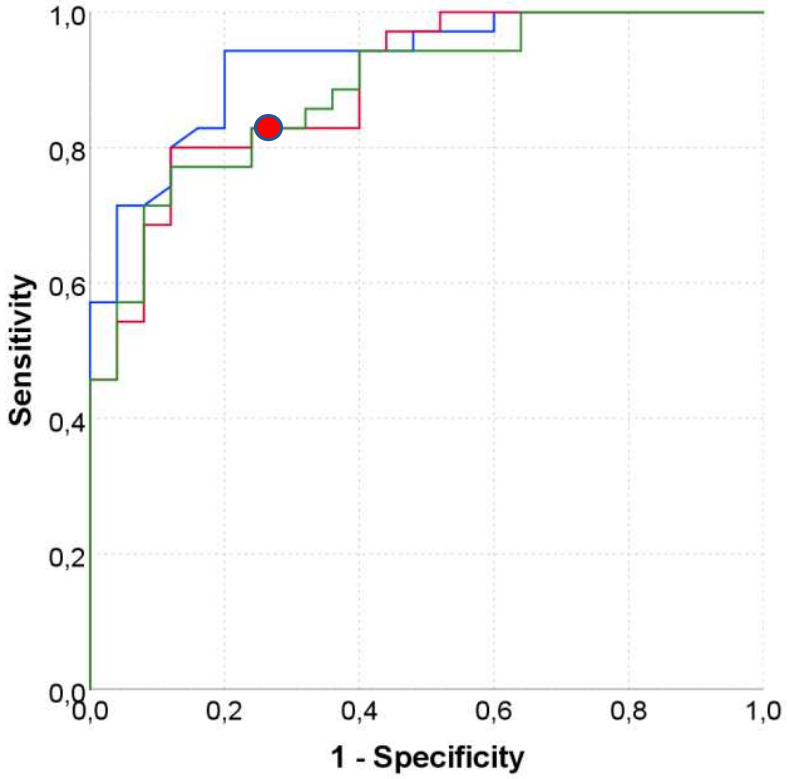

\section{Central device components}

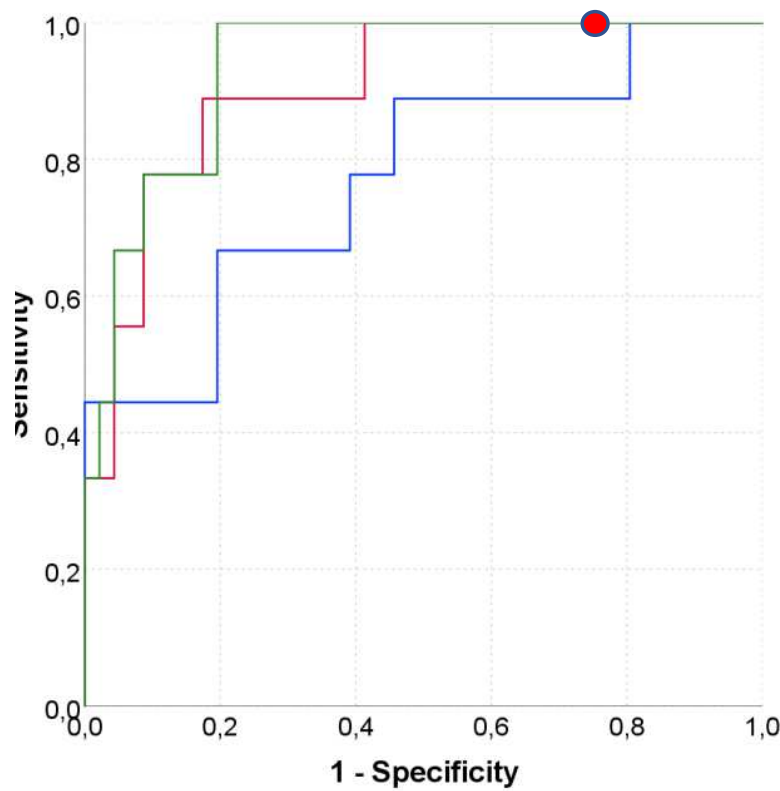

Legend:

Red dot: Visual analysis

\section{Blue line: SUVmax}

Red line: SUVratio (reference region bloodpool)

Green line: SUVratio (reference region liver)

\section{Optimum sensitivity / specificity:}

For suspected driveline infection:

SUVmax, cut-off 3.55, Se/Sp: 0.94 / 0.80

Visual analysis: $\mathrm{Se} / \mathrm{Sp}: 0.83 / 0.73$

For suspected central device component infection: SUVratio (bloodpool): Cut-off 3.04, Se/Sp: 0.89 / 0.83 SUVratio (liver): Cut-off 2.45, Se/Sp: $1.00 / 0.80$

Visual analysis: Se/Sp: 1.00 / 0.26 
Figure. 4: Flowchart FDG-PET/CT evaluation for DRI LVAD

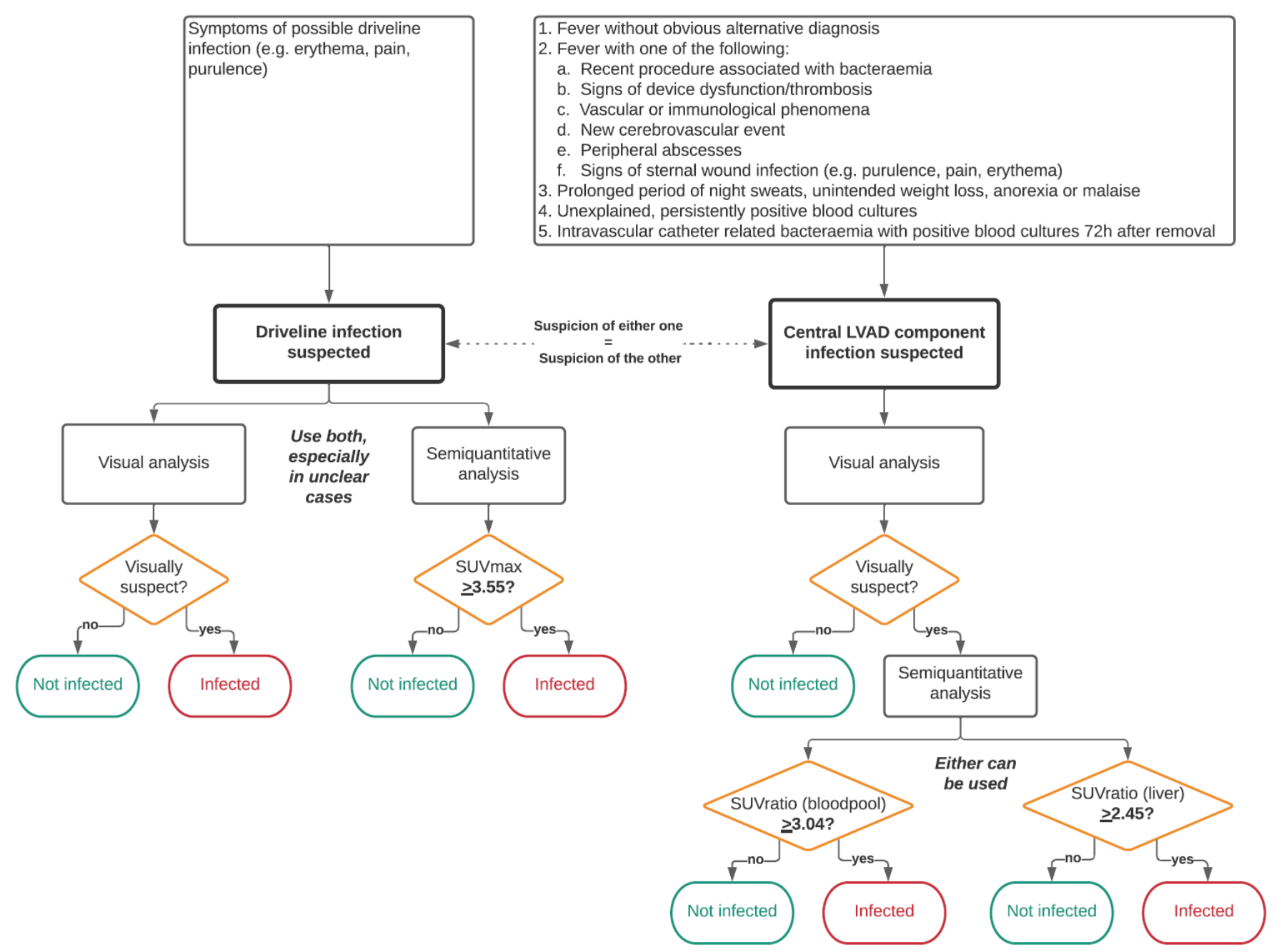

Legend: SUVmax: Maximum Standardized Uptake Value, SUVratio: Standardized Uptake Value Ratio 


\section{Supplementary Files}

This is a list of supplementary files associated with this preprint. Click to download.

- UnivariateanalysispotentialconfoundersLVADscans.xIsx 